PROCEEDINGS OF THE

AMERICAN MATHEMATICAL SOCIETY

Volume 135, Number 6, June 2007, Pages 1681-1688

S 0002-9939(07)08784-9

Article electronically published on February 9, 2007

\title{
VOLUME OF TRUNCATED FUNDAMENTAL DOMAINS
}

\author{
HENRY H. KIM AND LIN WENG
}

(Communicated by Wen-Ching Winnie Li)

\section{INTRODUCTION}

For $T \gg 0$, by applying Arthur's analytic truncation $\bigwedge^{T}$ to the constant function 1 on $G(F) \backslash G(\mathbb{A})^{1}$, we obtain a characteristic function of a compact subset $\mathfrak{F}(T)$ of $G(F) \backslash G(\mathbb{A})^{1}$. The aim of this paper is to give a general formula for the volume of $\mathfrak{F}(T)$, which is an exponential polynomial function of $T$. Consequently, by letting $T \rightarrow \infty$, we obtain a new way to evaluate the volume of fundamental domain for $G(F) \backslash G(\mathbb{A})^{1}$.

While $\mathfrak{F}(T)$ seems to be quite arbitrary, its volume admits beautiful structures:

(i) Surprisingly enough, the coefficients of $T$ are special values of zeta functions.

(ii) The volume is an alternating sum associated to standard parabolic subgroups. ((ii) is suggested by geometry — after all, $\mathfrak{F}(T)$ is obtained from $G(F) \backslash G(\mathbb{A})^{1}$ by truncating off cuspidal regions which are known to be parametrized by standard parabolic subgroups.)

Besides all basic properties of Arthur's analytic truncation, our method is based on a fundamental formula of Jacquet-Lapid-Rogawski on an integration of truncated Eisenstein series associated to cusp forms. Their formula, an advanced version of the Rankin-Selberg method, is obtained using the so-called Bernstein principle via regularized integration over cones.

\section{Arthur's trunchtion and integral of truncated Eisenstein SERIES}

Let $G$ be a reductive group and $P=M N$ be a parabolic subgroup. Fix a good maximal compact subgroup $K \subset G(\mathbb{A})$ such that the Iwasawa decomposition $G(\mathbb{A})=N(\mathbb{A}) M(\mathbb{A}) K$ holds. Then under suitable normalization of Haar measures, we have

$$
\int_{G(\mathbb{A})^{1}} f(g) d g=\int_{N(\mathbb{A})} \int_{A_{P}} \int_{M(\mathbb{A})^{1}} \int_{K} f(n a m k) e^{-2\left\langle\rho_{P}, H_{P}(a)\right\rangle} d n d a d m d k .
$$

An element $T \in \mathfrak{a}_{0}$ is said to be sufficiently positive and denoted by $T \gg 0$ if for all $\alpha \in \Delta_{0},\langle\alpha, T\rangle \gg 0$ are large enough. Fix such a $T$. Let $\phi: G(F) \backslash G(\mathbb{A}) \rightarrow \mathbb{C}$ be a smooth function. We define Arthur's analytic truncation $\bigwedge^{T} \phi$ (for $\phi$ with respect

Received by the editors March 22, 2006.

2000 Mathematics Subject Classification. Primary 11F72.

The first author was partially supported by an NSERC grant.

The second author was partially supported by a JSPS grant.

(C)2007 American Mathematical Society Reverts to public domain 28 years from publication 
to the parameter $T$ ) to be the function on $G(F) \backslash G(\mathbb{A})$ given by

$$
\left(\bigwedge^{T} \phi\right)(g):=\sum_{P: \text { standard }}(-1)^{\operatorname{dim}\left(Z_{P} / Z_{G}\right)} \sum_{\delta \in P(F) \backslash G(F)} \phi_{P}(\delta g) \cdot \hat{\tau}_{P}\left(H_{P}(\delta g)-T\right),
$$

where $\phi_{P}$ denotes the constant term of $\phi$ along with the standard parabolic subgroup $P, \hat{\tau}_{P}$ is the characteristic function of the so-called positive cone in $\mathfrak{a}_{P}$, and $H_{P}(g):=\log _{M} m_{P}(g)$ is an element in $\mathfrak{a}_{P}$. (Since $\hat{\tau}_{P}(\lambda)$ depends only on the projection $\lambda_{P}$ of $\lambda$ in $\mathfrak{a}_{P}$, it does not matter whether we view $H_{P}$ as a map onto $\mathfrak{a}_{P}$ or a map to $\mathfrak{a}_{0}$.)

Fundamental properties of Arthur's truncation may be summarized as

Theorem 1 ([A1, 2]). For a sufficiently positive $T$ in $\mathfrak{a}_{0}$, we have

(1) $\bigwedge^{T} \phi$ is rapidly decreasing, if $\phi$ is an automorphic form on $G(F) \backslash G(\mathbb{A})$,

(2) $\bigwedge^{T} \circ \bigwedge^{T}=\bigwedge^{T}$,

(3) $\bigwedge^{T}$ is self-adjoint,

(4) $\bigwedge^{T} \mathbf{1}$ is a characteristic function of a compact subset of $G(F) \backslash G(\mathbb{A})^{1}$.

Denote by $\mathfrak{F}(T)$ the compact subset of $G(F) \backslash G(\mathbb{A})^{1}$ whose characteristic function is given by $\bigwedge^{T} \mathbf{1}$ of (4).

Corollary 2. Let $T \gg 0$ be a fixed element in $\mathfrak{a}_{0}$. If $\phi$ is an automorphic form on $G(F) \backslash G(\mathbb{A})$,

$$
\int_{G(F) \backslash G(\mathbb{A})^{1}} \bigwedge^{T} \phi(g) d g=\int_{\mathfrak{F}(T)} \phi(g) d g .
$$

Proof. By (1), $\int_{G(F) \backslash G(\mathbb{A})^{1}} \bigwedge^{T} \phi(g) d g$ is well defined. Moreover,

$$
\begin{aligned}
& \int_{G(F) \backslash G(\mathbb{A})^{1}} \bigwedge^{T} \phi(g) d g \\
= & \int_{G(F) \backslash G(\mathbb{A})^{1}} \mathbf{1}(g) \cdot\left(\bigwedge^{T} \circ \bigwedge^{T}\right) \phi(g) d g \quad \text { (by (2) above) } \\
= & \int_{G(F) \backslash G(\mathbb{A})^{1}} \bigwedge^{T} \mathbf{1}(g) \cdot \bigwedge^{T} \phi(g) d g
\end{aligned}
$$

(by (3) above since $\bigwedge^{T} \phi(g)$ is rapidly decreasing)

$$
=\int_{G(F) \backslash G(\mathbb{A})^{1}}\left(\bigwedge^{T} \circ \bigwedge^{T}\right) \mathbf{1}(g) \cdot \phi(g) d g
$$

(by (3) again since $\phi$ is of moderate growth and $\bigwedge^{T} \mathbf{1}$ is compactly supported)

$$
\begin{array}{ll}
=\int_{G(F) \backslash G(\mathbb{A})^{1}} \bigwedge^{T} \mathbf{1}(g) \cdot \phi(g) d g & \text { (by (2) again) } \\
=\int_{\mathfrak{F}(T)} \phi(g) d g & \text { (by }(4)) .
\end{array}
$$

Recall the following formula of Jacquet-Lapid-Rogawski.

Theorem 3 ([JLR, Corollary 17]). Let $P=M N$ be a parabolic subgroup and let $(M, \sigma)$ be a cuspidal datum. Let $E(g, \varphi, \lambda)$ be an Eisenstein series where $\varphi \in$ 
$\mathcal{A}_{P}(G)_{\sigma}$. Then

$$
\int_{G(F) \backslash G(\mathbb{A})^{1}} \bigwedge^{T} E(g, \varphi, \lambda) d g
$$

is equal to zero if $P$ is not a minimal parabolic. If $P$ is minimal, it is equal to

$$
v \sum_{w \in \Omega} \frac{e^{\langle w \lambda-\rho, T\rangle}}{\prod_{\alpha \in \Delta_{0}}\left\langle w \lambda-\rho, \alpha^{\vee}\right\rangle} \int_{M(F) \backslash M(\mathbb{A})^{1} \times K} M(w, \lambda) \varphi(m k) d m d k,
$$

where $v=\operatorname{Vol}\left(\left\{\sum_{\alpha \in \Delta_{0}} a_{\alpha} \alpha^{\vee}: 0 \leq a_{\alpha}<1\right\}\right)$.

Here the volume $v$ appears because the measure on $\mathfrak{a}_{0}$ is defined using co-weights $\left\{\varpi_{\alpha}^{\vee}: \alpha \in \Delta_{0}\right\}$. So $v$ is equal to the order of the fundamental group of $\Phi[\mathrm{Hu}$, page 68] for simply laced groups. For example, $v=n$ for $S L_{n} ; v=2$ for $E_{7}, v=4$ for $D_{l}, v=3$ for $E_{6}$.

\section{MAIN RESULT}

Now we consider the special case of the $G$ split, semi-simple group, $\varphi=1$ : The constant term of $E(g, 1, \lambda)$ along the Borel subgroup is given by

$$
E_{B}(g, 1, \lambda)=\sum_{w \in \Omega} m(w, \lambda) e^{\langle w \lambda+\rho, H(g)\rangle}
$$

where $\rho=\rho_{B}$, and

$$
m(w, \lambda)=\prod_{\alpha>0, w \alpha<0} \frac{\xi_{F}\left(\left\langle\lambda, \alpha^{\vee}\right\rangle\right)}{\xi_{F}\left(\left\langle\lambda, \alpha^{\vee}\right\rangle+1\right)},
$$

by the Gindikin-Karpelevich formula. Here $\xi_{F}$ is the Dedekind zeta function with the usual $\gamma$-factors, namely, $\xi_{F}(s)=\left(2^{-2 r_{2}} \pi^{-N} d_{F}\right)^{\frac{s}{2}} \Gamma\left(\frac{s}{2}\right)^{r_{1}} \Gamma(s)^{r_{2}} \zeta_{F}(s)$, where $\zeta_{F}(s)$ is the Dedekind zeta function, $[F: \mathbb{Q}]=N=r_{1}+2 r_{2}$, and $d_{F}$ is the discriminant. Also $r_{1}\left(r_{2}\right)$ is the number of real (complex) places of $F$, resp.

Then $\zeta_{-1}=\operatorname{res}_{s=1} \zeta_{F}(s)=\operatorname{Vol}\left(F^{*} \backslash \mathbf{I}_{F}^{1}\right)=\frac{2^{r_{1}}(2 \pi)^{r_{2}} h R}{w d_{F}^{\frac{1}{2}}}$, where $h$ is the class number, $R$ the regulator, and $w$ the number of roots of unity in $F$. We see that

$$
\xi_{-1}=\operatorname{res}_{s=1} \xi_{F}(s)=\frac{2^{r_{1}} h R}{w}=(2 \pi)^{-r_{2}} d_{F}^{\frac{1}{2}} \cdot \operatorname{Vol}\left(F^{*} \backslash \mathbf{I}_{F}^{1}\right) .
$$

Hence we have

$$
\begin{aligned}
\int_{G(F) \backslash G(\mathbb{A})^{1}} & \bigwedge^{T} E(g, 1, \lambda) d g \\
= & v \sum_{w \in \Omega} \frac{e^{\langle w \lambda-\rho, T\rangle}}{\prod_{\alpha \in \Delta_{0}}\left\langle w \lambda-\rho, \alpha^{\vee}\right\rangle} m(w, \lambda) \int_{M(F) \backslash M(\mathbb{A})^{1}} d m,
\end{aligned}
$$

since $e^{\langle w \lambda+\rho, H(m)\rangle}=1$ for $m \in M(\mathbb{A})^{1}$. We look at the residue at $\lambda=\rho$. Langlands La] showed that the residue of $E(g, 1, \lambda)$ at $\lambda=\rho$ is constant. Hence the residue is given by the residue of the constant term along the Borel subgroup. Note that $\left\langle\rho, \alpha^{\vee}\right\rangle=1$ for $\alpha \in \Delta_{0}$ and $\left\langle\rho, \alpha^{\vee}\right\rangle>1$ if $\alpha \notin \Delta_{0}, \alpha>0$. Hence the residue of $E(g, 1, \lambda)$ at $\lambda=\rho$ is given by

$$
\operatorname{res}_{\lambda=\rho} E(g, 1, \lambda)=\lim _{\lambda \rightarrow \rho} \prod_{\alpha \in \Delta_{0}}\left(\left\langle\lambda, \alpha^{\vee}\right\rangle-1\right) \cdot m\left(w_{0}, \lambda\right)
$$


where $w_{0}$ is the longest Weyl group element, i.e.,

$$
m\left(w_{0}, \lambda\right)=\prod_{\alpha>0} \frac{\xi_{F}\left(\left\langle\lambda, \alpha^{\vee}\right\rangle\right)}{\xi_{F}\left(\left\langle\lambda, \alpha^{\vee}\right\rangle+1\right)} .
$$

For $i>1$, let $n_{i}=\#\left\{\alpha>0:\left\langle\rho, \alpha^{\vee}\right\rangle=i\right\}-\#\left\{\alpha>0:\left\langle\rho, \alpha^{\vee}\right\rangle=i-1\right\}$. Then

$$
\operatorname{res}_{\lambda=\rho} E(g, 1, \lambda)=\xi_{-1}^{r} \cdot \prod_{i>1} \xi_{F}(i)^{n_{i}},
$$

where $r$ is the rank of $G$.

Now by Corollary 2,

$$
\int_{G(F) \backslash G(\mathbb{A})^{1}} \bigwedge^{T} E(g, 1, \lambda) d g=\int_{\widetilde{F}(T)} E(g, 1, \lambda) d g .
$$

Hence

$$
\begin{aligned}
\operatorname{res}_{\lambda=\rho}\left(\int_{G(F) \backslash G(\mathbb{A})^{1}} \bigwedge^{T} E(g, 1, \lambda) d g\right) & =\int_{\mathfrak{F}(T)} \operatorname{res}_{\lambda=\rho} E(g, 1, \lambda) d g \\
& =\operatorname{Vol}(\mathfrak{F}(T)) \cdot \operatorname{res}_{\lambda=\rho} E(g, 1, \lambda) .
\end{aligned}
$$

So the residue at $\lambda=\rho$ of the left-hand side of $(*)$ is

$$
\xi_{-1}^{r} \prod_{i>1} \xi_{F}(i)^{n_{i}} \cdot \operatorname{Vol}(\mathfrak{F}(T)) .
$$

On the other hand, the residue at $\lambda=\rho$ of the right-hand side of $(*)$ is

$$
\operatorname{Vol}\left(T(F) \backslash T(\mathbb{A})^{1}\right) \cdot \lim _{\lambda \rightarrow \rho}\left(v \sum_{w \in \Omega} e^{\langle w \lambda-\rho, T\rangle} m(w, \lambda) \prod_{\alpha \in \Delta_{0}} \frac{\left\langle\lambda, \alpha^{\vee}\right\rangle-1}{\left\langle w \lambda, \alpha^{\vee}\right\rangle-1}\right) .
$$

Here $\operatorname{Vol}\left(T(F) \backslash T(\mathbb{A})^{1}\right)=\left(\operatorname{Vol}\left(F^{*} \backslash \mathbf{I}_{F}^{1}\right)\right)^{r}=\zeta_{-1}^{r}$. Clearly $\left\langle w \lambda, \alpha^{\vee}\right\rangle=\left\langle\lambda,\left(w^{-1} \alpha\right)^{\vee}\right\rangle$. Also given $w \in \Omega$, the sets $\left\{\alpha \in \Delta_{0}: w \alpha<0\right\}$ and $\left\{\alpha \in \Delta_{0}: w \alpha \in \Delta_{0}\right\}$ are disjoint. Let $W_{0}$ be the set of all $w \in \Omega$ such that

$$
\left\{\alpha \in \Delta_{0}: w \alpha<0\right\} \cup\left\{\alpha \in \Delta_{0}: w \alpha \in \Delta_{0}\right\}=\Delta_{0} .
$$

Let $r_{w}=\#\left\{\alpha \in \Delta_{0}: w \alpha<0\right\}$. For $i>1$, let

$$
n_{i, w}=\#\left\{\alpha>0, w \alpha<0:\left\langle\rho, \alpha^{\vee}\right\rangle=i\right\}-\#\left\{\alpha>0, w \alpha<0:\left\langle\rho, \alpha^{\vee}\right\rangle=i-1\right\} .
$$

Then

$$
\begin{aligned}
\lim _{\lambda \rightarrow \rho}\left(\sum_{w \in \Omega} e^{\langle w \lambda-\rho, T\rangle} m(w, \lambda)\right. & \left.\prod_{\alpha \in \Delta_{0}} \frac{\left\langle\lambda, \alpha^{\vee}\right\rangle-1}{\left\langle w \lambda, \alpha^{\vee}\right\rangle-1}\right) \\
& =\sum_{w \in W_{0}} e^{\langle w \rho-\rho, T\rangle} \frac{\xi_{-1}^{r_{w}} \prod_{i>1} \xi_{F}(i)^{n_{i, w}}}{\prod_{\alpha \in \Delta_{0}, w^{-1} \alpha \notin \Delta_{0}}\left(\left\langle w \rho, \alpha^{\vee}\right\rangle-1\right)} .
\end{aligned}
$$

Note that $w=1 \in W_{0}$ and it gives rise to 1 . Also $w=w_{0} \in W_{0}$ and it gives rise to $(-2)^{-r} \cdot \xi_{-1}^{r} \prod_{i>1} \xi_{F}(i)^{n_{i}} \cdot e^{\langle-2 \rho, T\rangle}$.

Therefore,

$$
\begin{aligned}
\operatorname{Vol}(\mathfrak{F}(T))= & v(2 \pi)^{r_{2} r} d_{F}^{-\frac{r}{2}} \prod_{i>1} \xi_{F}(i)^{-n_{i}} \\
& \cdot \sum_{w \in W_{0}} \xi_{-1}^{r_{w}} \frac{\prod_{i>1} \xi_{F}(i)^{n_{i, w}}}{\prod_{\alpha \in \Delta_{0}, w^{-1} \alpha \notin \Delta_{0}}\left(\left\langle w \rho, \alpha^{\vee}\right\rangle-1\right)} \cdot e^{\langle w \rho-\rho, T\rangle} .
\end{aligned}
$$


We claim that there is one to one correspondence between $W_{0}$ and the standard parabolic subgroups of $G$. The correspondence is as follows: Given $w \in W_{0}$, let $J_{w}=\left\{\alpha \in \Delta_{0}: w \alpha \in \Delta_{0}\right\}$. Let $P=P_{w}$ be the standard parabolic subgroup corresponding to $J_{w}$.

Conversely, let $J \subset \Delta_{0}$. Then there is the set $D_{J}$ of distinguished coset representatives of $W / W_{J}$. The elements in $D_{J}$ are characterized by the property $w J>0$. Let $w_{J}$ be the element of the maximal length in $D_{J}$. Let $w_{l}$ be the element of the maximal length in $W$, and let $w_{l, J}$ be the element of the maximal length in $W_{J}$. Then $w_{J}=w_{l} w_{l, J}$, and $W_{0}=\left\{w_{J}: J \subset \Delta\right\}$ [Ca, Prop. 1.14].

We claim that if $w \in W_{0},\left\langle w \rho, \alpha^{\vee}\right\rangle<0$ for all $\alpha \in \Delta_{0}-w J_{w}$ : Suppose that for $\alpha \in \Delta_{0}-w J_{w}, w_{l}(\alpha)=-\beta$ for some $\beta \in \Delta_{0}$. Since $w^{-1}=w_{l, J_{w}} w_{l}, w^{-1}(\alpha)=$ $-w_{l, J_{w}}(\beta)$. If $\beta \in J_{w}$, then $-w_{l, J_{w}}(\beta) \in J_{w}$ and $w^{-1}(\alpha) \in J_{w}$. But since $\alpha \notin w J_{w}$, $w^{-1}(\alpha) \notin J_{w}$. Contradiction. Hence $\beta \notin J_{w}$ and $w_{l, J_{w}}>0$. Therefore $w^{-1}(\alpha)<0$. Hence

$$
\prod_{\alpha \in \Delta_{0}, w^{-1} \alpha \notin \Delta_{0}}\left(\left\langle w \rho, \alpha^{\vee}\right\rangle-1\right)=(-1)^{\mathrm{rank} P_{w}} \prod_{\alpha \in \Delta_{0}-w J_{w}}\left(1-\left\langle w \rho, \alpha^{\vee}\right\rangle\right) .
$$

Theorem 4. Let $\mathfrak{F}(T)$ be the truncated domain for a sufficiently positive $T$. Then

$$
\begin{aligned}
\operatorname{Vol}(\mathfrak{F}(T))=v \cdot(2 \pi)^{r_{2} r} d_{F}^{-\frac{r}{2}} \prod_{i>1} \xi_{F}(i)^{-n_{i}} \\
\qquad \sum_{w \in W_{0}}(-1)^{r a n k P_{w}} \xi_{-1}^{r_{w}} \frac{\prod_{i>1} \xi_{F}(i)^{n_{i, w}}}{\prod_{\alpha \in \Delta_{0}-w J_{w}}\left(1-\left\langle w \rho, \alpha^{\vee}\right\rangle\right)} \cdot e^{\langle w \rho-\rho, T\rangle} .
\end{aligned}
$$

Note that as $T \rightarrow \infty$, only the term corresponding to $w=1$ survives and the limit is exactly the volume of the fundamental domain given by Langlands [La, namely,

Corollary 5 (Langlands). If $\mathfrak{F}$ is the fundamental domain, then

$$
\operatorname{Vol}(\mathfrak{F})=v \cdot(2 \pi)^{r_{2} r} d_{F}^{-\frac{r}{2}} \cdot \prod_{i>1} \xi_{F}(i)^{-n_{i}} .
$$

We note that if $w \in W_{0}, r_{w}+\sum n_{i, w}=0$, and $-\sum n_{i}=r$, the rank of $G,-\sum n_{i}$ is the number of positive roots such that $\left\langle\rho, \alpha^{\vee}\right\rangle=1$. It is exactly $r$. Similarly, $-\sum n_{i, w}$ is the number of positive roots such that $w \alpha<0$, and $\left\langle\rho, \alpha^{\vee}\right\rangle=1$. It is exactly $r_{w}=\operatorname{rank} P_{w}$. So $r_{w}+\sum\left(-n_{i}+n_{i, w}\right)=r$ for each $w \in W_{0}$. Then we can write the formula in Theorem 4 as

Corollary 6. Let $\mathfrak{F}(T)$ be the truncated domain for a sufficiently positive $T$. Then

$$
\begin{aligned}
& \operatorname{Vol}(\mathfrak{F}(T)) \\
& \quad=v \cdot(2 \pi)^{r_{2} r} d_{F}^{-\frac{r}{2}} \cdot \sum_{J \subset \Delta_{0}}(-1)^{r a n k P_{J}} \frac{\xi_{-1}^{r a n k P_{J}} \prod_{i>1} \xi_{F}(i)^{-n_{i}+n_{i, w_{J}}}}{\prod_{\alpha \in \Delta_{0}-w_{J} J}\left(1-\left\langle w_{J} \rho, \alpha^{\vee}\right\rangle\right)} \cdot e^{\left\langle w_{J} \rho-\rho, T\right\rangle} .
\end{aligned}
$$

Remark. The formula, as it stands, suggests that volumes of cuspidal regions corresponding to parabolic subgroups can be written in terms of special zeta values as well.

We look at three examples. 
Example 1. $G=S p_{4}$. Let $\Delta_{0}=\left\{e_{1}-e_{2}, 2 e_{2}\right\}$. There are 4 positive roots $\left\{e_{1} \pm\right.$ $\left.e_{2}, 2 e_{1}, 2 e_{2}\right\}$ and 8 Weyl group elements $\left\{1,(12), c_{1}, c_{2},(12) c_{1},(12) c_{2},(12) c_{1} c_{2}, c_{1} c_{2}\right\}$, where $c_{i}$ 's are sign changes. Then $\rho=2 e_{1}+e_{2}$. In this case, $\left\{e_{1}, e_{1}+e_{2}\right\}$ is a basis of the co-weight lattice. Let

$$
T=x \cdot e_{1}+y \cdot\left(e_{1}+e_{2}\right), \quad x, y \gg 0 .
$$

Then there are 4 standard parabolic subgroups corresponding to

$$
J_{1}=\Delta_{0}, \quad J_{c_{1}}=\left\{2 e_{2}\right\}, \quad J_{(12) c_{1} c_{2}}=\left\{e_{1}-e_{2}\right\}, \quad J_{c_{1} c_{2}}=\emptyset .
$$

Accordingly, $v=1$, and

$$
\begin{aligned}
\operatorname{Vol}(\mathfrak{F}(T)) & =(2 \pi)^{2 r_{2}} d_{F}^{-1}\left(\xi_{F}(2) \xi_{F}(4)\right. \\
& \left.-\frac{1}{4} \cdot \xi_{-1} \xi_{F}(2) \cdot e^{-4 x-4 y}-\frac{1}{3} \cdot \xi_{-1} \xi_{F}(2) \cdot e^{-3 x-6 y}+\frac{1}{4} \cdot \xi_{-1}^{2} \cdot e^{-4 x-3 y}\right) .
\end{aligned}
$$

Example 2. $G=S L_{4}$. Let $\Delta_{0}=\left\{e_{1}-e_{2}, e_{2}-e_{3}, e_{3}-e_{4}\right\}$. There are 6 positive roots and 24 Weyl group elements. Here $\rho=\frac{1}{2}\left(3 e_{1}+e_{2}-e_{3}-3 e_{4}\right)$, and $\left\{\frac{1}{4}\left(3 e_{1}-e_{2}-e_{3}-e_{4}\right), \frac{1}{2}\left(e_{1}+e_{2}-e_{3}-e_{4}\right), \frac{1}{4}\left(e_{1}+e_{2}+e_{3}-3 e_{4}\right)\right\}$ is a basis of the co-weight lattice. Let

$T=x \cdot \frac{1}{4}\left(3 e_{1}-e_{2}-e_{3}-e_{4}\right)+y \cdot \frac{1}{2}\left(e_{1}+e_{2}-e_{3}-e_{4}\right)+z \cdot \frac{1}{4}\left(e_{1}+e_{2}+e_{3}-3 e_{4}\right)$,

with $x, y, z \gg 0$. Then there are 8 standard parabolic subgroups corresponding to

$$
\begin{gathered}
J_{1}=\Delta_{0}, \quad J_{(1432)}=\left\{e_{2}-e_{3}, e_{3}-e_{4}\right\}, \quad J_{(13)(24)}=\left\{e_{1}-e_{2}, e_{3}-e_{4}\right\}, \\
J_{(1234)}=\left\{e_{1}-e_{2}, e_{2}-e_{3}\right\}, \quad J_{(1423)}=\left\{e_{3}-e_{4}\right\}, \quad J_{(14)}=\left\{e_{2}-e_{3}\right\}, \\
J_{(1324)}=\left\{e_{1}-e_{2}\right\}, \quad J_{(14)(23)}=\emptyset .
\end{gathered}
$$

Accordingly, $v=4$, and

$$
\begin{aligned}
\operatorname{Vol}(\mathfrak{F}(T)) & =4 \cdot(2 \pi)^{3 r_{2}} d_{F}^{-\frac{3}{2}} \cdot\left(\xi_{F}(2) \xi_{F}(3) \xi_{F}(4)-\frac{1}{4} \cdot \xi_{-1} \xi_{F}(2) \xi_{F}(3) \cdot e^{-x-2 y-3 z}\right. \\
- & \frac{1}{4} \cdot \xi_{-1} \xi_{F}(2)^{2} \cdot e^{-2 x-4 y-2 z}-\frac{1}{4} \cdot \xi_{-1} \xi_{F}(2) \xi_{F}(3) \cdot e^{-3 x-2 y-z} \\
& +\frac{1}{6} \cdot \xi_{-1}^{2} \xi_{F}(2) \cdot e^{-2 x-4 y-3 z}+\frac{1}{9} \cdot \xi_{-1}^{2} \xi_{F}(2) \cdot e^{-3 x-3 y-3 z} \\
& \left.+\frac{1}{6} \cdot \xi_{-1}^{2} \xi_{F}(2) \cdot e^{-3 x-4 y-2 z}-\frac{1}{8} \cdot \xi_{-1}^{3} \cdot e^{-3 x-4 y-3 z}\right)
\end{aligned}
$$

Example 3. More generally, let $G=\mathrm{SL}_{n}$. Let $\Delta_{0}=\left\{e_{1}-e_{2}, \ldots, e_{n-1}-e_{n}\right\}$. In this case, $n_{i}=-1$ for $i=2, \ldots, n ; \rho=\frac{1}{2}\left((n-1) e_{1}+(n-3) e_{2}+\cdots-(n-1) e_{n}\right)$. Any parabolic subgroup is associated to a parabolic subgroup with the Levi subgroup whose derived group is $\mathrm{SL}_{m_{1}} \times \cdots \times \mathrm{SL}_{m_{k}}$, where $m_{1}+\cdots+m_{k}+m=n$. We assume that $m_{1} \geq \cdots \geq m_{k} \geq 2$ and $k \geq 2$. It corresponds to the subset

$$
\begin{array}{r}
J=\left\{e_{1}-e_{2}, \ldots, e_{m_{1}-1}-e_{m_{1}}, e_{m_{1}+1}-e_{m_{1}+2}, \ldots, e_{m_{1}+m_{2}-1}-e_{m_{1}+m_{2}}, \ldots,\right. \\
\left.e_{m_{1}+\cdots+m_{k-1}+1}-e_{m_{1}+\cdots+m_{k}}\right\} .
\end{array}
$$


Then $\operatorname{rank} P_{J}=m+k-1$, and $w_{J}$ is given by the combination of

$$
\begin{aligned}
& \left(\begin{array}{ccc}
1 & \cdots & m_{1} \\
n-m_{1}+1 & \cdots & n
\end{array}\right) \quad\left(\begin{array}{ccc}
m_{1}+1 & \cdots & m_{1}+m_{2} \\
n-m_{1}-m_{2}+1 & \cdots & n-m_{1}
\end{array}\right) \\
& \left(\begin{array}{ccc}
m_{1}+\cdots+m_{k-1}+1 & \cdots & m_{1}+\cdots+m_{k-1}+m_{k} \\
m+1 & \cdots & m+m_{k}
\end{array}\right) \quad\left(\begin{array}{ccc}
n-m+1 & \cdots & n \\
m & \cdots & 1
\end{array}\right) \text {. }
\end{aligned}
$$

We can show that

$$
\begin{aligned}
\Delta_{0}-w_{J} J=\left\{e_{1}-e_{2}, \ldots, e_{m}-e_{m+1}, e_{n-m_{1}}-e_{n-m_{1}+1}, \ldots,\right. \\
\left.e_{n-m_{1}-\cdots-m_{k-1}}-e_{n-m_{1}-\cdots-m_{k-1}+1}\right\},
\end{aligned}
$$

and $n_{i, W_{J}}$

$$
= \begin{cases}k-1, & \text { for } 1 \leq i \leq m_{k}, \\ k-j, & \text { for } m_{k-j+2}+\cdots+m_{k}+1 \leq i \leq m_{k-j+1}+\cdots+m_{k}, \\ & \text { and } 2 \leq j \leq k \\ -1, & \text { for } m_{1}+\cdots+m_{k}<i \leq n .\end{cases}
$$

Hence if $m \geq 1$, the coefficient corresponding to $J$ in Corollary 6 is

$$
\frac{(-1)^{m+k-1}}{2^{m-1}\left(m_{1}+m_{2}\right)\left(m_{2}+m_{3}\right) \cdots\left(m_{k-1}+m_{k}\right)\left(m_{k}+1\right)} \cdot\left(\xi_{-1}^{m+k-1} \prod_{j=1}^{k} \prod_{i=2}^{m_{j}} \xi_{F}(i)\right) \text {. }
$$

If $m=0$, then it is

$$
\frac{(-1)^{k-1}}{\left(m_{1}+m_{2}\right)\left(m_{2}+m_{3}\right) \cdots\left(m_{k-1}+m_{k}\right)} \cdot\left(\xi_{-1}^{k-1} \prod_{j=1}^{k} \prod_{i=2}^{m_{j}} \xi_{F}(i)\right) .
$$

\section{ACKNOWLEDGMEnTs}

This work was done while the second author was visiting the University of Toronto in the fall of 2005. He would like to thank the Department of Mathematics for its hospitality and Professors James Arthur and Henry Kim for their kind invitation.

\section{REFERENCES}

[A1] J. Arthur, A trace formula for reductive groups II. Applications of a truncation operator, Compositio Math. 40 (1980), 87-121. MR0558260 (81b:22018)

[A2] , A measure on the unipotent variety, Canad. J. Math 37 (1985), 1237-1274. MR:0828844 (87m:22049)

[Ca] W. Casselman, Introduction to the Theory of Admissible Representations of p-adic Reductive Groups, unpublished notes.

[Hu] J. Humphreys, Introduction to Lie Algebras and Representation Theory, Springer-Verlag, 1972. MR 0323842 (48:2197)

[JLR] H. Jacquet, E. Lapid and J. Rogawski, Periods of automorphic forms, J. of AMS 12 (1999), 173-240. MR:1625060 (99c:11056)

[La] R.P. Langlands, The volume of the fundamental domain for some arithmetical subgroups of Chevalley groups, in Algebraic Groups and Discontinuous Subgroups, Proc. Sympos. Pure Math. 9, 1966, pp. 143-148. MR0213362(35:4226) 
Department of Mathematics, University of Toronto, Toronto, Ontario M5S 2E4 And - Korea Institute for Advanced Study, Seoul, Korea

E-mail address: henrykim@math.toronto.edu

Graduate School of Mathematics, Kyushu University, Fukuoka, Japan

E-mail address: weng@math.kyushu-u.ac.jp 